\title{
Study on building electricity consumption data collection methods Ling Cao ${ }^{1, a}$, Pei Gang Liu ${ }^{2, b}$ and Nian Yan Huang ${ }^{2, c}$
}

${ }^{1}$ School of software engineering, Chongqing University of Posts and Telecommunications, chongqing 400065, China;

${ }^{2}$ School of software engineering, Chongqing University of Posts and Telecommunications, chongqing 400065, China.

acaoling@cqupt.edu.cn, b1032802604@qq.com, c597785047@qq.com

Keywords: High Stability, Acquisition Protocol, Breakpoint Transmition, AES encryption

\begin{abstract}
The stability of high energy consumption data acquisition system in addition to the traditional energy consumption data acquisition, processing and upload function, but also on the acquisition of communication protocol is analyzed and improved, to improve the accuracy of the data. Design breakpoint transmition to ensure data continuity, through the MD5 checksum AES encryption application design and enhance the security of data upload, provide accurate data services for the public building energy-saving emission reduction.
\end{abstract}

\section{Introdution}

Large public buildings energy waste is very serious, there is a huge saving space. Traditional bulky data acquisition systems, real-time poor, severe signal attenuation, a limited number of plug-in devices. With the improvement of building energy consumption monitoring system for building energy consumption data acquisition requirements are also getting higher and higher. So this paper embedded development technology, C \# programming language and VS2008 realized energy collector software features. Focus on energy consumption data collection protocol, energy security data processing method and data upload to do a more detailed study.

\section{Research on energy consumption data acquisition and improvement of communication protocols}

Electricity meters comply with industry standard DL / T 645-1997 "multi-function meter communication protocol", it found that many traditional acquisition system resolution protocol is incorrect. Traditional data collection often lost count, the number of error affect the reliability of the measurement results, the measurement may lead to serious results completely unavailable.

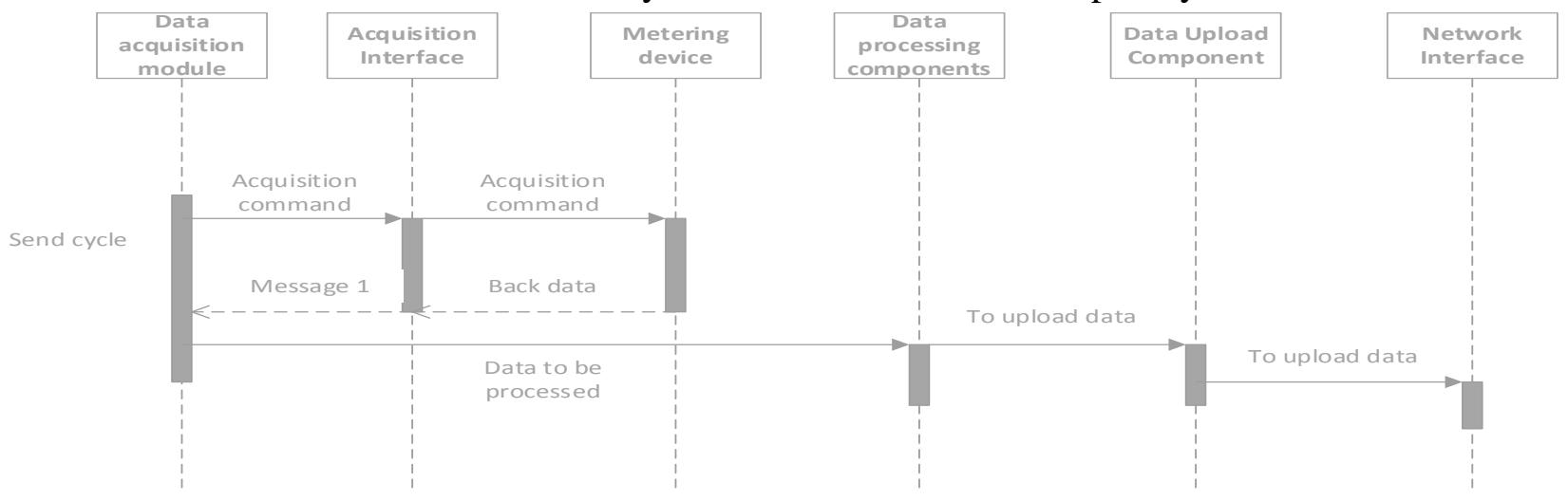

\subsection{Drain the case frame}

Fig. 1 The overall design timing diagram

The previous data check using only CS code check. There may be no data leakage analysis frame. Check on the frame data acquisition module to increase the. To determine whether to accept a complete data frame to solve the problem of lost data. 


\subsection{Lack of "zero" phenomenon}

Parsing the data field should be processed in accordance with the agreement plus $33 \mathrm{H}$. Parsed data 123456.78 , when the "34" to "04", often to 0 to miss. The original data will be parsed $120,456.7812,456.78$ data vary greatly. Increase the frame data acquisition components complement "zero." Analyzing data is greater than 10, if not preceded by 0 .

\subsection{Serial parse error}

Serial class SerialPort, DataReceive event (event data receiving method) does not guarantee that every byte received initiation DataReceived event. Custom serial class uses SerialPort1. Determine the amount of data remaining in the buffer to be read by BytesToRead property. When receiving data from the SerialPort object, again cited DataReceived event on the secondary thread.

\section{The data processing method of energy consumption}

\subsection{Breakpoint Transmition}

When the power off or network outages, restart to reconnect to the network after the acquisition system can resume normal work and query XML breakpoints and resume broken net storage of energy consumption data. The data acquisition system in the XML file upload batch and type marking distinction. Upload component receives the report data according to reported data processing procedures, when receives the HTTP data is called HTTP data method and after all the data upload then call report data processing method.

When the network is smooth, resume need continuous upload multiple XML documents, which requires all data upload completed is send to the collector to confirm the information. The continuous method defined two integer variables total and current. As long as current=total showed complete resume. Analysis of $\mathrm{Xml}$ document, database connection to send data packets to the server.

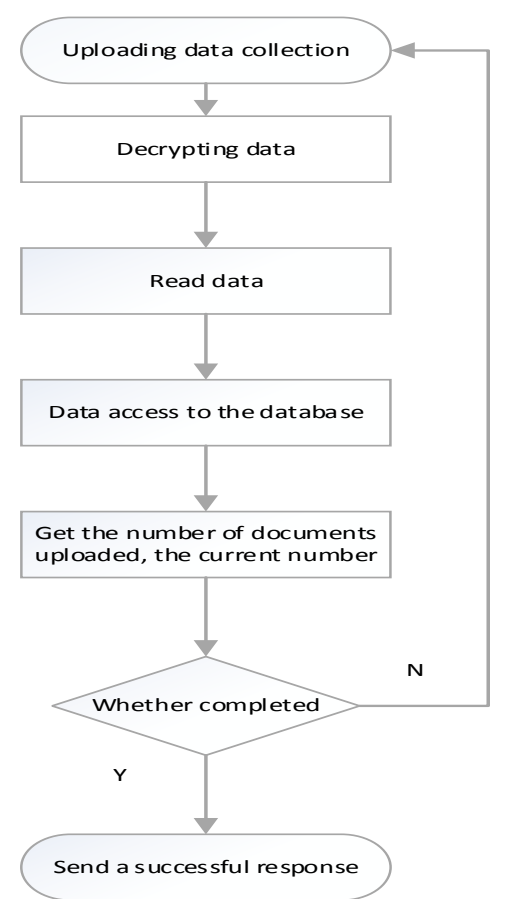

Fig. 2 Flowchat of breakpoint transmition

\subsection{A method of processing data packets}

A large amount of data easily lead to data loss. Contraction flow using GZipStream compression and decompression. The GZip data format of such industry standard algorithm it uses lossless compression and decompression file. This format includes a cyclic redundancy check value of damage detection data. The main purpose of the XML file compression to reduce network traffic load and prevent data loss rate. About seventy percent can reduce the file size. 


\section{MD5 checksum AES encryption design and Application}

According to the requirements issued by the Ministry of housing and urban rural development, the state organ office buildings and large public building energy consumption monitoring system sub energy data transmission technology guidelines for that data before uploading, data center will sync, working mode detection work of data acquisition gateway authentication, heartbeat. According to the requirements of authentication process need to do MD5 encryption, AES encryption need to upload the data before. The data acquisition system to upload data when the traditional TCP transmission protocol is not only used for authentication and encryption is easy to attack and steal data. Design of MD5 verification and AES algorithm is used to improve the safety and reliability of energy consumption data acquisition system.

\subsection{MD5 program design verification}

MD5 in the acquisition system mainly includes two parts: one part to complete the user password encryption; the other part of the authentication device. By a solid state key authentication and dynamic key. The server must generate random sequences for acquisition system calculation.

\subsection{The research and design of encryption AES}

The AES algorithm is mainly used for remote data stream encryption with encryption and decryption functions. The upload component specifically for AES encryption and decryption algorithm into a type of packaging. The basic is divided into three parts: the generation of encryption and decryption key scheduling table, obtain the plaintext and ciphertext output. The use of AES encryption algorithm for XML packet encryption, key length is 128bit. The local file system encryption key is stored in the data center and data acquisition, data center can update the data collector through the network encryption key.

\section{Summary}

Through proper parsing of communication protocols and improved data collection and research MD5 checksum AES encryption design application store HTTP methods. Improved data collection accuracy, timeliness, security. The current trend of building is intelligent, green technology, which is environmentally sustainable and resource requirements of the development results. High stability of the energy consumption data acquisition systems provide reliable data for the energy consumption monitoring platform for energy conservation in existing buildings, to establish energy consumption quotas and over-quota increases the system lay the foundation for the promotion of high value and economic benefits.

\section{Reference}

[1] Huangjun Peng, Li Zhengrong. EEB Computer Evaluation System [J]. HVAC, 2004,47 (11).

[2] Klaus Wehrle,Frank Pehlke. The Linux Networking Architecture:Design and Implementation of Network [M].Upper Saddle River:Prentice Hall,2004.

[3] San Disk Corporation. San Disk MultiMediaCard Product Manual.Version 2.0,2004.

[4] Louati, Onay Erkose, Zeghlache. An XML-based configuration management architecture for IP routers.Proceedings of the IEEE International Conference on Electronics, Circuits, and Systems.NJ:IEEE,2005:1-4 .

[5] SQLite Docunmentation.The Architecture of SQLite,2008,12.

[6] Gradara J S.Investigating large software system evolution: the Linux kemel.Proceedings of 26 Annual International Computer Software and Application Conference,2002.

[7] Wang Tengjiao, Yang Dongqing, Tang Shiwei. Discovering and materialized XML views in data integration system. Database Engineering and Applications Symposium,NJ:IEEE.2004,1.

[8] STM32F4xx on http://www.st.com. 